\title{
Sternectomy for Candida albicans sternal osteomyelitis after left ventricular assist device implantation
}

\author{
Mathias Van Hemelrijck ${ }^{1}$ - Michelle Frank ${ }^{2}$ - Annelies S. Zinkernagel ${ }^{3} \cdot$ Ronny Buechel $^{4}$ - Juri Sromicki ${ }^{1}$. \\ Markus J. Wilhelm ${ }^{1} \cdot$ Holger Klein ${ }^{5} \cdot$ Barbara Hasse $^{3} \cdot$ Carlos A. Mestres $^{1}$
}

Received: 4 November 2020 / Revised: 30 December 2020 / Accepted: 6 January 2021/Published online: 17 February 2021

(C) The Author(s) 2021

\begin{abstract}
Fungal osteomyelitis is an uncommon complication after cardiac surgery and associated with high mortality. A case of Candida albicans and Staphylococcus epidermidis osteomyelitis with device infection after implantation of a left ventricular assist device in a 60-year-old male patient is presented here. After clinical identification and confirmation with microbiological examinations and fluorodeoxyglucose positron emission tomography (FDG-PET) scan, debridement was performed. Surgical specimens grew C. albicans and S. epidermidis. Fluconazole, daptomycin, and negative pressure wound therapy were initiated, but failed to achieve healing. Total sternectomy and pectoralis flap reconstruction were performed. There was no recurrent infection for C. albicans on a prolonged antifungal regime. The combination of antifungal therapy and aggressive surgical debridement may be useful to control fungal osteomyelitis.
\end{abstract}

Keywords Left ventricular assist device $\cdot$ Endocarditis $\cdot$ Sternectomy $\cdot$ Fungal osteomyelitis

\section{Introduction}

Fungal osteomyelitis is a serious albeit uncommon condition after cardiac surgery. It requires aggressive therapy and is associated with high mortality. Treatment strategy includes prolonged antifungal therapy and surgical debridement, whereas the extent of excision is still a matter of debate. A case of Candida albicans and coagulase-negative staphylococci sternal osteomyelitis and concomitant left ventricular assist device (LVAD) infection, its therapy, and outcome are discussed.

Carlos A. Mestres

Carlos.Mestres@usz.ch

1 Clinic for Cardiac Surgery, University Hospital Zurich, Rämistrasse 100, CH-8091 Zürich, Switzerland

2 Clinic for Cardiology, University Hospital Zurich, Zurich, Switzerland

3 Division of Infectious Diseases and Hospital Epidemiology, University Hospital Zurich, Zurich, Switzerland

4 Department of Nuclear Medicine, Cardiac Imaging, University Hospital Zurich, Zurich, Switzerland

5 Department of Plastic Surgery and Hand Surgery, University Hospital Zurich, Zurich, Switzerland

\section{Case report}

A 60-year-old male patient underwent LVAD (HeartWare ${ }^{\circledR}$ ) implantation in November 2017 as a bridge-to-transplantation due to ischemic cardiomyopathy. He required 6 mediastinal re-explorations due to recurrent bleeding. The revision procedures performed in this patient were always categorized by the operating surgeons as diffuse bleeding without surgical origin. The patient was given multiple coagulation factors, including Factor VIII, due to the suspicion of LVAD-associated von Willebrand disease. After bleeding was controlled, he subsequently was discharged from the hospital 2 months postoperatively. Three months later, he was readmitted due to pulmonary decompensation and sternal wound infection (SWI). Fluorodeoxyglucose positron emission tomography (FDGPET) scan confirmed sternal uptake; the full report confirmed high suspicion of strongly metabolically active sternal infection with small retrosternal abscess formation. There was mild reactive lymphadenopathy, mediastinal and axillary on both sides, and only moderately increased metabolic activity around the LVAD, which could be due to artifacts. There were slightly metabolically active infiltrates in the dorso-basal areas of the right lower lobe with suspected fibrotic changes after pneumonia. Otherwise, no further infection foci were detected (Fig. 1). Surgical debridement of the sternum was performed. 


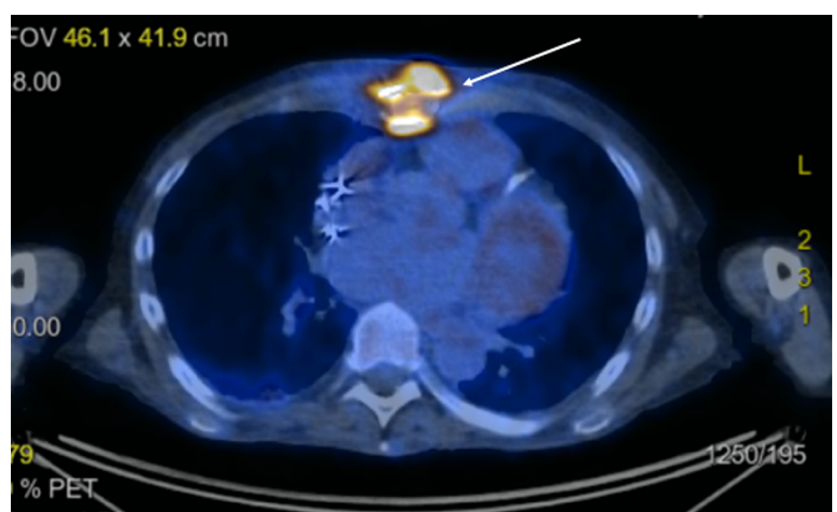

Fig. 1 Sternal uptake in a fluorodeoxyglucose positron emission tomography (FDG-PET) scan

C. albicans and Staphylococcus epidermidis grew in the operative samples. Attention was then paid to the sternal region as per clinical signs and FDG-PET information as described. Negative pressure wound therapy (NPWT) was initiated. NPWT lasted 3 months, until the wound was closed. The antimicrobial therapy included caspofungin $50 \mathrm{mg} / 24 \mathrm{~h}$ IV and daptomycin $10 \mathrm{mg} / \mathrm{kg}$ body weight $/ 24 \mathrm{~h} \mathrm{IV}$. No attempt to work with antifungal and or antibiotic-impregnated crystals/granules was made, due to the lack of supporting evidence. Sequential samples of sternal tissue, taken during repetitive wound revisions, were persistently positive for C. albicans and S. epidermidis. Blood cultures were negative, and a subsequent FDG-PET scan suggested persistent sternal infection and a new abscess-like formation around the LVAD. We scheduled the patient for surgical resection and dead space filling with an adjacent muscle flap. LVAD exchange was not an option, since the new device would have had to be implanted in an already infected area. Heart transplantation could not be offered to the patient due to his poor condition. LVAD explantation was not an option because of severely reduced left ventricular function. Total sternectomy and pectoralis muscle flap closure of the chest were successfully performed (Fig. 2). After a complicated in-hospital course, which included pneumonia requiring temporary veno-venous extracorporeal membrane oxygenation (ECMO) and add-on of an antimicrobial therapy with meropenem $2 \times 1 \mathrm{~g}$ /day i.v. (nosocomial pneumonia) and clarithromycin $2 \times 500 \mathrm{mg} /$ day p.o. No LVAD dysfunction was noticed at any time after sternectomy and muscle-plasty chest closure. The patient was discharged after almost 9 months in the hospital.

Six months later, under suppressive antifungal therapy with oral fluconazole $400 \mathrm{mg} / 24 \mathrm{~h}$, no signs of fungal growth could be observed. However, a mechanical skin perforation above the LVAD outflow cannula was documented and subsequent debridement with LVAD coverage by a myo-cutaneous latissimus dorsi muscle flap was performed (Fig. 3). Cultures of the excised ulcerated tissue and all collected blood cultures did not show fungal or antimicrobial growth; furthermore, aspergillus antigen was negative (index 0.04). After an initially satisfactory postoperative course, the patient developed bilateral pneumonia and died 9 days after flap coverage. Postmortem examination disclosed respiratory failure with diffuse alveolar damage as the immediate cause of death. The pathological examination confirmed onset of acute bronchopneumonia of the right lower lobe. No pathogen was detected in special and immunohistochemical stains, especially for fungi or viruses. Numerous iron-laden intra-alveolar macrophages; fibrosis demarcated pleural empyema on the left $(12 \times 6 \times 6 \mathrm{~cm})$; trachea with subglottic scarring (max. $1.3 \mathrm{~cm}$, probably post-intubation); pleural adhesions on both sides; and fibrinous pleurisy with no pleural effusion were noticed. In essence, further findings showed a chronic leftsided pleural empyema with pus surrounding the LVAD driveline. However, there were no persistent signs of osteomyelitis. Summarizing, the first revision for deep sternal
Fig. 2 a Mediastinum after sternectomy: the arrow points to the caudal portion of the outflow cannula. b Pectoralis flap covering the mediastinum
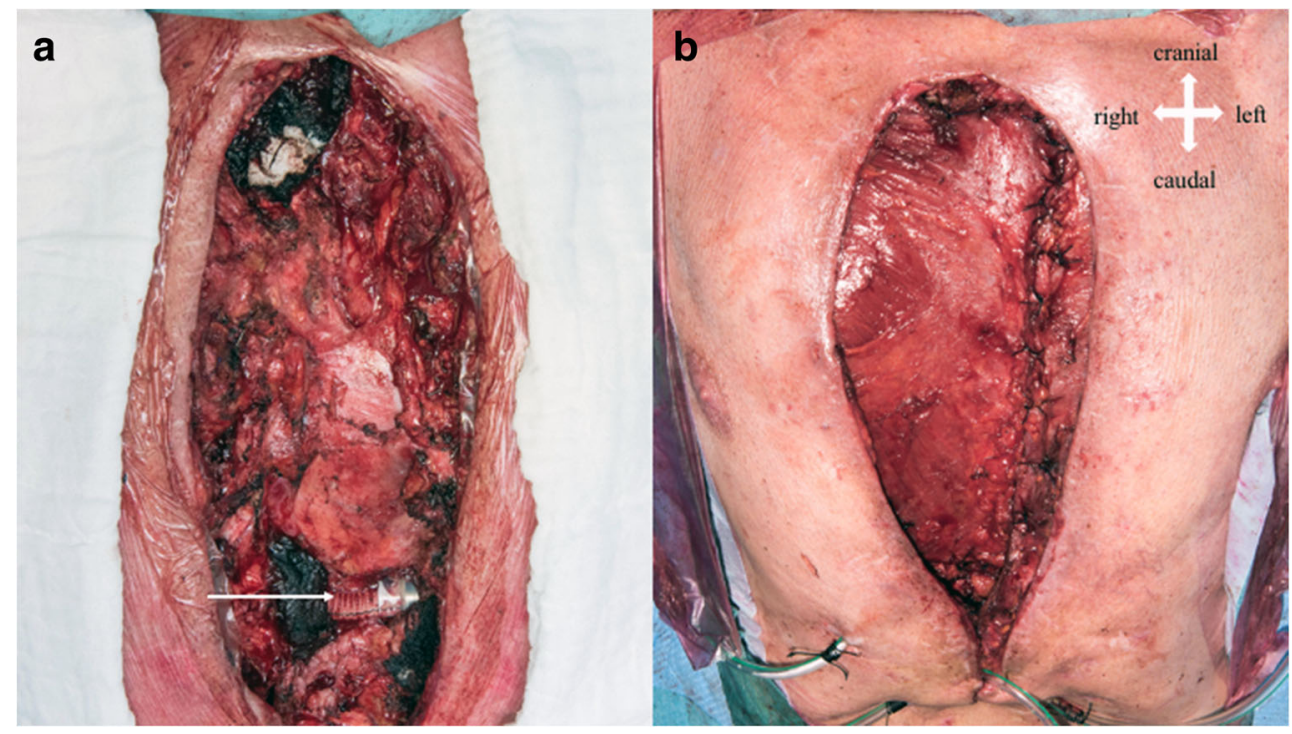
Fig. 3 Latissimus dorsi muscle flap: a before incision, $\mathbf{b}$ after skin demarcation; $\mathbf{c}$ before covering the mediastinum and left ventricular assist device (circle) with a latissimus dorsi muscle flap (star); $\mathbf{d}$ final result. The arrow points in all images to the mediastinum

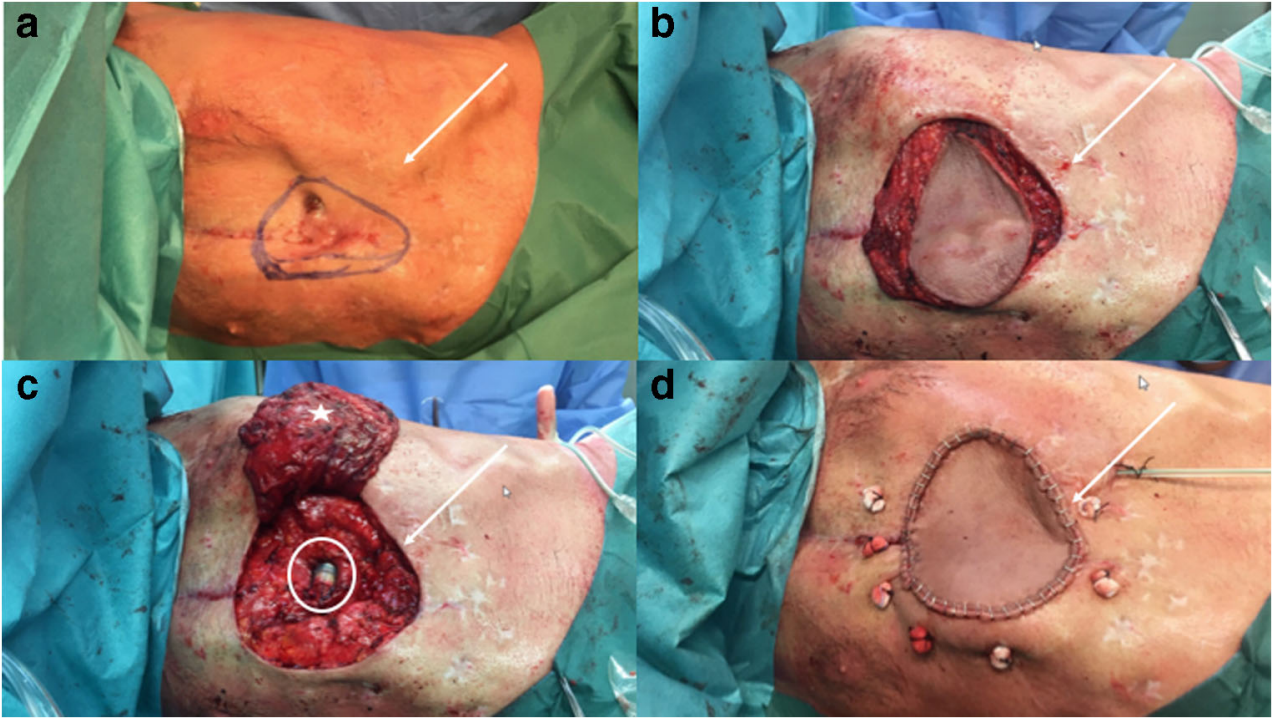

wound infection (DSWI) was performed 6 months after LVAD implantation. Sternectomy was finally performed 5 months after the first debridement and 11 months after LVAD implantation. Overall, the patient died from hospitalacquired pneumonia 20 months after the LVAD was implanted.

\section{Comment}

Deep sternal wound infection after cardiac surgery has an incidence of around 2\% [1], with a reported mortality of $55 \%$ [2]. Candida osteomyelitis represents a severe and uncommon condition that requires combined surgical debridement and prolonged antifungal treatment. Re-sternotomies due to non-infective causes, prolonged use of antibiotics, colonization of the respiratory and urinary tracts, and the use of percutaneous dilatational tracheostomy devices have been identified as risk factors facilitating Candida infections [2]. Fungal osteomyelitis represents a treatment challenge, for which a number of surgical strategies have been suggested [1-3]. The optimal treatment strategy is still not clear $[2,3]$. There are no significant outcome differences between the different surgical approaches, although more promising results have been confirmed with omental flaps in a recent review by Abu-Omar and colleagues [3]. But omental flaps require the opening of the abdominal cavity, harshly increasing the patient's mortality. The use of a NPWT and a muscle flap is recommended by the European Association of Cardiothoracic Surgery (EACTS) as class I and IIb recommendation, respectively, with a level of evidence $\mathrm{B}$ in DSWI. However, there is no clear treatment strategy regarding fungal osteomyelitis [3]. On the other hand, Pappas et al. recommend surgical debridement and an antifungal regime of 6 to 12 months [4]. These recommendations are based on case reports and small series $[5,6]$. In terms of timing of secondary wound closure, there is still a gap of knowledge among current guidelines and recommendation reports.

The implicated patient suffered from a combination of DSWI and LVAD-associated infection. In a recent multicenter study, Tattevin and colleagues reported $30 \%$ of infections after LVAD implantation, with $4 \%$ being cannula or pump related. Candida-associated LVAD infections were found in $6 \%$ of the patients. In this prospective study, the mortality rate among infected patients was $10 \%$ [7]. Regarding treatment strategies, it has been suggested that persistent infections under a suppressive antibiotic regime might require device explantation and subsequent heart transplantation [8]. In the case reported herein, an initial debridement with NPWT was unsuccessful. The patient therefore underwent total sternectomy and muscle flap closure. Since neither device explantation nor heart-transplantation was possible in our case, we decided that long-term suppressive antifungal therapy was mandatory. We achieved control of fungal osteomyelitis in this exceedingly uncommon case of infection in a patient under long-term mechanical circulatory support.

Author contributions All authors contributed to the study conception and design. Material preparation, and data collection were performed by Mathias Van Hemelrijck, Barbara Hasse and Carlos A. Mestres. The first draft of the manuscript was written by Mathias Van Hemelrijck and all authors commented on previous versions of the manuscript. All authors read and approved the final manuscript.

Funding Open access funding provided University of Zurich.

\section{Compliance with Ethical Standards}

Conflict of interest The authors have no relevant financial or nonfinancial interests to disclose.

Informed Consent Informed consent (general consent) was collected and signed before the submission of this document. This document is in accordance with the ethical standards. 
Consent for publication Informed consent (general consent) was collected and signed before the submission of this document. According to local regulations, no further signed document was required for the submission of this work.

Open Access This article is licensed under a Creative Commons Attribution 4.0 International License, which permits use, sharing, adaptation, distribution and reproduction in any medium or format, as long as you give appropriate credit to the original author(s) and the source, provide a link to the Creative Commons licence, and indicate if changes were made. The images or other third party material in this article are included in the article's Creative Commons licence, unless indicated otherwise in a credit line to the material. If material is not included in the article's Creative Commons licence and your intended use is not permitted by statutory regulation or exceeds the permitted use, you will need to obtain permission directly from the copyright holder. To view a copy of this licence, visit http://creativecommons.org/licenses/by/4.0/.

\section{References}

1. Modrau IS, Ejlertsen T, Rasmussen BS. Emerging role of Candida in deep sternal wound infection. Ann Thorac Surg. 2009;88:1905-9.
2. Arikan AA, Omay O, Kanko M, et al. Treatment of Candida sternal infection following cardiac surgery - a review of literature. Infect Dis. 2019;51:1-11.

3. Abu-Omar Y, Kocher GJ, Bosco P, et al. European Association for Cardio-Thoracic Surgery expert consensus statement on the prevention and management of mediastinitis. Eur J Cardiothorac Surg. 2017;51:10-29.

4. Pappas PG, Kauffman CA, Andes DR, et al. Clinical practice guideline for the management of candidiasis: 2016 update by the Infectious Diseases Society of America. Clin Infect Dis. 2016;62: e1-50.

5. Clancy CJ, Nguyen MH, Morris AJ. Candidal mediastinitis: an emerging clinical entity. Clin Infect Dis. 1997;25:608-13.

6. Malani PN, McNeil SA, Bradley SF, Kauffman CA. Candida albicans sternal wound infections: a chronic and recurrent complication of median sternotomy. Clin Infect Dis. 2002;35:1316-20.

7. Tattevin P, Flecher E, Auffret V, et al. Risk factors and prognostic impact of left ventricular assist device-associated infections. Am Heart J. 2019;214:69-76.

8. O'Horo JC, Abu Saleh OM, Stulak JM, Wilhelm MP, Baddour LM, Sohail MR. Left ventricular assist device infections: a systematic review. ASAIO J. 2018;64:287-94.

Publisher's note Springer Nature remains neutral with regard to jurisdictional claims in published maps and institutional affiliations. 